\title{
The Role of Follow-up in Monitoring the Outcomes of Prematurity in a Cohort of Romanian Infants
}

\author{
Anca Bivoleanu1, Andreea Avasiloaiei2, Mihaela Moscalu³, Maria Stamatin2 \\ ${ }^{1}$ Regional Neonatal Intensive Care Unit, Grigore T. Popa University of Medicine and Pharmacy, Cuza-Voda Clinical Hospital of Obstetrics and Gynaecology, Iasi, \\ Romania \\ ${ }^{2}$ Regional Neonatal Intensive Care Unit, Division of Neonatology, Department of Mother and Child Care, Grigore T. Popa University of Medicine and Pharmacy, \\ Cuza-Voda Clinical Hospital of Obstetrics and Gynaecology, Iasi, Romania \\ ${ }^{3}$ Department of Preventive Medicine and Interdisciplinary Sciences, Grigore T. Popa University of Medicine and Pharmacy, Iasi, Romania
}

Background: The rate of preterm births in Romania is one of the highest among European countries. However, there is little information regarding the ways in which premature birth affects the outcome in Romanian preterm infants.

Aims: To investigate the effects of early developmental intervention after discharge from the hospital on motor and cognitive development in preterm infants.

Study Design: Longitudinal observational study

Methods: We performed the Amiel-Tison neurologic evaluation at discharge and the Bayley Scales of Infant Development from 3 to 24 months. Based on these evaluations, an outcome score was formulated.

Results: Between 2007 and 2010, 1157 of 2793 premature infants were included into the study. There was a negative correlation between the number of evaluations and the risk of developing neurologic sequelae $(p<0.001)$. The correlation analysis demonstrated a significant association between the final category of risk at the end of the follow up program and the degree of compliance $(p<0.01)$. At 24 months evaluation, there was a correlation between the low gestational age and the risk of developing severe neurologic sequelae $(\mathrm{p}<0.001)$.

Conclusion: This study shows the importance of follow up program in decreasing the risk of developing neurologic sequelae in preterm infants.

Keywords: Neurologic sequelae, prematurity, infants, monitoring
Prematurity involves complex medical conditions (mainly respiratory, but also infectious, gastrointestinal, feeding related and growth related), in addition to neurodevelopmental morbidities (neurologic, motor, sensory and behavioral), the severity of which increases with decreasing gestational age. These conditions affect the risk of morbidity and mortality, and they significantly influence later quality of life (1-4). 1 in 10 premature infants will develop a permanent minor neurological dysfunction, such as language disorders, learning disabilities, attention deficit-hyperactivity disorder, neuro-motor dysfunction or developmental coordination disorders, behavioral problems, and social-emotional difficulties, but also chronic health issues such as asthma, infections and increased risk of Sudden Infant Death Syndrome (5-9).

Due to injury to the developing brain, $50 \%$ of premature infants born before the 26th week of gestation may present

\footnotetext{
Address for Correspondence: Dr. Andreea Avasiloaiei, Regional Neonatal Intensive Care Unit, Division of Neonatology, Department of Mother and Child Care, Grigore T. Popa University of Medicine and Pharmacy, Cuza-Voda Clinical Hospital of Obstetrics and Gynaecology, Iasi, Romania

Phone: +40744830367_e-mail: andreea.avasiloaiei@umfiasi.ro

Received: 27 August $2015 \quad$ Accepted: 25 January 2016 $\bullet$ DOI: 10.4274/balkanmedj.2015.1125

Available at www.balkanmedicaljournal.org

Cite this article as:

Bivoleanu A, Avasiloaiei A, Moscalu M, Stamatin M. The role of follow-up in monitoring the outcomes of prematurity in a cohort of romanian infants. Balkan Med J 2017;34:21-7

${ }^{\circ}$ Copyright 2017 by Trakya University Faculty of Medicine / The Balkan Medical Journal published by Galenos Publishing House.
} 
major neurological sequelae (e.g., cerebral palsy (CP), mental retardation, visual and hearing deficits) and minor issues (e.g, impaired cognitive abilities and physical skills, learning difficulties, depression, anxiety, or difficulty interacting with other children their age) (10-13). Therefore, it is essential for follow-up services to provide medical, developmental evaluation and support for this category of patients.

In Romania, the rate of preterm births is $9 \%$, almost twice as high as in the majority of European countries $(14,15)$. Because in our country little information is available regarding the ways in which premature birth affects outcome, we sought to estimate the role of follow-up programs and early developmental intervention in evaluating the degree of risk for neurologic sequelae in a cohort of premature infants from our Maternity Hospital.

\section{MATERIALS AND METHODS}

\section{Study population}

This longitudinal observational study was conducted on 1157 preterm infants from January 1, 2007 to December 31, 2010 at a tertiary-level Neonatal Intensive Care Unit in Iasi, Romania. Inclusion criteria were in accordance with our national guidelines for follow-up of high-risk neonates (16): preterm birth, birth weight of less than 1500 grams, perinatal asphyxia with Apgar scores of 3 or less at 5 minutes and/or signs of hypoxic-ischemic encephalopathy, mechanical ventilation for more than 24 hours, infants with central nervous system injury (intraventricular hemorrhage, periventricular leukomalacia, seizures, hydrocephalus, encephalopathy), extracranial or intracranial trauma, respiratory disorders, including bronchopulmonary dysplasia, necrotizing enterocolitis, infections-meningitis and/or culture positive sepsis, hyperbilirubinemia that requires exchange transfusion, fetal growth restriction, multiple preterm births, metabolic issuessymptomatic hypoglycemia and hypocalcemia, abnormal neurological examination at discharge, social/environmental conditions (low economic status, underage mothers, drugs/ alcohol abuse, smoking).

Infants with major congenital anomalies and infants requiring major surgery were excluded. Perinatal data were collected prospectively during admission, stored in the neonatal intensive care unit database, and retrospectively retrieved for data analysis. Parental informed consent was obtained for participation in the follow-up program and inclusion in the present study. The hospital's Committee of Ethics approved of our research.

\section{Survey protocol}

At discharge, or as close as possible to the postmenstrual age of 40 weeks, the neonatal neurological examination was performed according to the Amiel-Tison protocol, by the same trained neonatologist, who examined all infants included in this study. The Amiel-Tison test $(17,18)$ involves 35 items covering neurosensory aspects, cranial morphology, passive and active muscle tones, spontaneous motor activity and primary reflexes. The scoring system is based on a three-point ordinal scale where ' 0 ' corresponds to a typical response, ' 1 ' to a moderately abnormal response, and ' 2 ' to a definitely abnormal response. Items that have neuropsychological relevance include: neurosensory aspects (alertness, visual fixation), cranial morphology (head circumference, anterior fontanels, squamous sutures and other sutures), passive tone in limbs and axis, rapid stretching, active tone and primitive reflexes. The infants were placed in three risk categories [low (L), moderate (M), severe (S)], based on the number of abnormal examination findings.

At 3, 6, 9, 12, 18 and 24 months corrected age, we performed the Bayley Scales of Infant Development, 2nd Edition (BSID II) (19-21). All developmental milestones are assessed according to corrected age, in order to compensate for prematurity. The results were summarized into five domains: cognitive development, neuromotor development, language development, behavior and general health. During regular evaluations, according to total number of items failed, we placed the infants in three risk categories (L, M, S). If the infant had on-going issues or illnesses, such as delays in reaching developmental milestones, we recommended more frequent visits and physiotherapy. Mothers were also instructed to perform their infants' specific daily exercises. As needed, the patient was referred to another specialist for early intervention services and/or to recommend further evaluation and therapy.

According to the total number of items failed during regular evaluations and at the end of the follow-up program, we included the infants in specific risk categories: L, M or S.

We considered the patient to be low risk if the patient presented with minor hypotonia or hypertonia without other neurologic abnormalities (cognitive and learning deficits, behavioral and emotional abnormalities) and/or minor language delay. M risk was defined as mild deviation in muscle tone regulation, reflexes, fine or gross motor performance, moderate cognitive and/or speech or language disorders. Major risk was defined as severe neurodevelopment dysfunctions: $\mathrm{CP}$, mental retardation, visual impairment and/or hearing loss. For this study, we chose gestational age, birth weight, respiratory distress syndrome, intracranial hemorrhage, and periventricular leukomalacia in order to examine the correlation between these entities and the category of risk.

Because the infants with regular evaluations were referred to special rehabilitation programs, in order to correlate compliance 
with the follow-up program and risk categories (L/M/Saccording to the BSID II examination), we created an "outcome score", as follows:

" 0 " if the infant at the final evaluation, at two years corrected age, maintained the same risk category, (L/M/S)

"1", if the infant at the final evaluation, progressed from moderate to L risk or from $\mathrm{S}$ to $\mathrm{M}$,

" 2 " for those infants, who passed at the final evaluation directly from $\mathrm{S}$ risk to L risk,

"-1" if the infant at the final evaluation, regressed from L risk to $\mathrm{M}$ and/or from $\mathrm{M}$ to $\mathrm{S}$ risk,

"-2" for patients who, at the final evaluation, passed directly from $\mathrm{L}$ to $\mathrm{S}$ risk.

\section{Statistical analysis}

The data were analyzed using SPSS version 20.0 (SPSS Inc.; Chicago, IL, USA). Data were expressed as mean, standard deviation (SD), median, and interquartile range, or as per cent frequency, as appropriate. Comparisons among groups were made by $\mathrm{p}$ value for linear trends (1-way analysis of variance or chi-square test). Among patients, comparisons were made by paired t test (for normally distributed data) or by Wilcoxon signed rank test (for non-normally distributed data). Correlations between variables were investigated using the Pearson product moment correlation coefficient (r), contingency coefficient, or by Spearman Rank correlation coefficient, as appropriate. Statistical significance was defined as $\mathrm{p}<0.05$.

\section{RESULTS}

Over the four-year period of this study, 4116 infants were admitted to our hospital. $67.9 \%(n=2793)$ were premature and $41.4 \%(n=1157)$ were enrolled in the follow-up program and in this study. $2.4 \%(n=28)$ of patients died after discharge and before completing the follow-up program-2 years corrected age. The percentage of premature infants among those admitted to the hospital increased during the study period, from $19.3 \%$ in 2007 , to $27.2 \%$ in 2010 (Figure 1).

Mean gestational age was $31.9( \pm 2.0 \mathrm{SD})$ weeks. The frequency of infants with gestational age less than 34 weeks decreased significantly, while the frequency of infants with gestational age over 34 weeks increased during the studied period $(\mathrm{F}=7.45$, $\mathrm{p}=0.00006,95 \% \mathrm{CI})$. The mean birth weight was $1720( \pm 541$ SD) grams.

When evaluated by the Amiel-Tison neurologic examination tool at discharge, $47.3 \%$ of infants were found to be L risk; 41.7\% were $\mathrm{M}$ risk; $11.1 \%$ were $\mathrm{S}$ risk.

Mothers/families were informed about the significance and importance of the follow-up program and were asked to return for periodic evaluations according to a personalized schedule. $72.7 \%(n=841)$ came for all 6 recommended evaluations, $20.3 \%$ $(n=235)$ came $2-5$ times for evaluation and $7.0 \%(n=81)$ came for only one examination, and therefore their results were analyzed based only according to this single evaluation. Of those with six evaluations, the highest percentage was represented by infants of gestational age 34-36 weeks: $42.0 \%$. The results indicate a significant association between gestational age of infants and the frequency of evaluations during the follow-up program $\left(\chi^{2}=36.014, r=0.4627, p=0.00247,95 \% \mathrm{CI}\right)$.

At every stage of the follow-up evaluation, we changed the respective category of risk according to the infant's performance. The highest percentage of infants with severe $(8.7 \%)$ and medium risk $(62.8 \%)$ was at three month corrected age (Table 1). The rate of death correlated significantly with low gestational age, as proven by the non-parametric analysis $\left(\chi^{2}=18.64, r=0.508, p=0.00317,95 \%\right.$ CI $)$.

According to the BSID II evaluation, at the end of the follow-up program, 3.6\% were included in the high-risk group, and 26.3\% were placed in the medium-risk group; $68.5 \%$ were considered to have a L risk of developing subsequent neurologic disabilities. Premature infants that presented for all six recommended evaluations made up the highest percentage of infants at $\mathrm{L}$ risk (88.1\%), as compared to those with a single evaluation $(27.2 \%)$. The present study highlights a significant correlation between the number of evaluations and the risk in developing neurological sequelae $\left(\chi^{2}=35.19, \mathrm{r}=0.709, \mathrm{p}=0.000142,95 \%\right.$ CI) (Figure 2).

The association between gestational age and the degree of estimated risk observed at the end of the follow-up program demonstrates a significant correlation between low gestational age and a $\mathrm{S}$ risk of developing neurological sequelae $\left(\chi^{2}=27.008\right.$, $\mathrm{r}=0.629, \mathrm{p}=0.00014,95 \% \mathrm{CI}$ ) (Figure 3). All patients with $\mathrm{S}$ risk at discharge and only one evaluation were deceased.

Regarding the infants who attended all six evaluations, for the high-risk category, according to the Amiel-Tison examination,

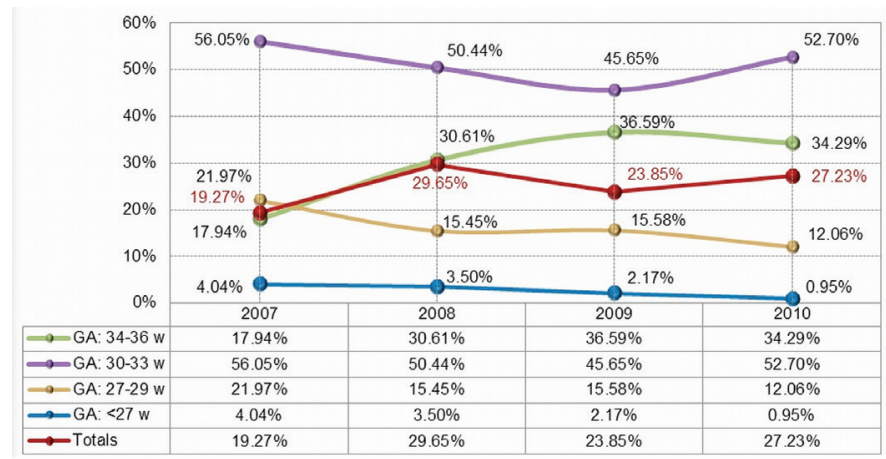

FIG. 1. The incidence of prematurity among study subjects. GA: gestational age 
$68.0 \%$ passed from $\mathrm{S}$ risk to $\mathrm{M} ; 3.0 \%$ passed from $\mathrm{S}$ risk $\mathrm{t} \mathrm{L}$ risk; $2.9 \%$ remained in the $\mathrm{S}$ risk category.

Among those with $\mathrm{M}$ risk, 27.1\% remained in this category, $72.4 \%$ reached $\mathrm{L}$ risk and only $0.5 \%$ moved into the S risk category. Furthermore, $97.1 \%$ of patients found with L risk at discharge remained in this category, and only $2.9 \%$ passed into $\mathrm{M}$ risk (Figure 4). There was a significant correlation between the result of the final examination at 2 years corrected age and the Amiel-Tison examination and the frequency of evaluations $\left(\chi^{2}=339.3, \mathrm{p}<0.01\right)$.

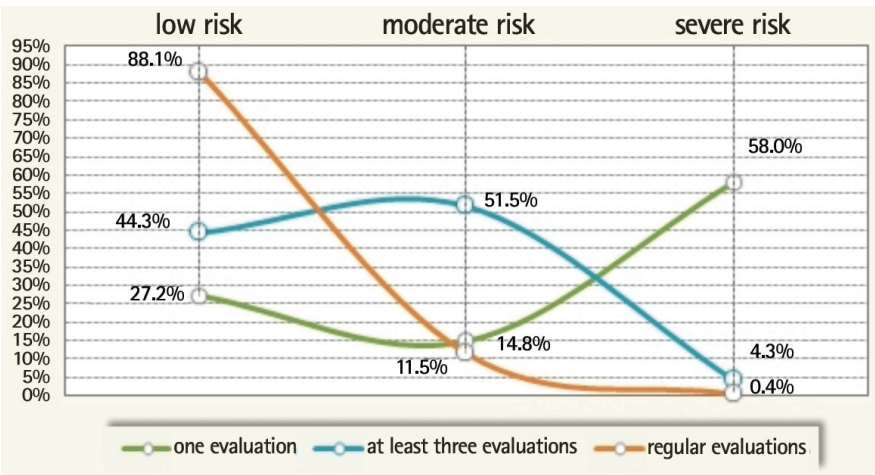

FIG. 2. Correlation between the degree of risk and number of evaluations.

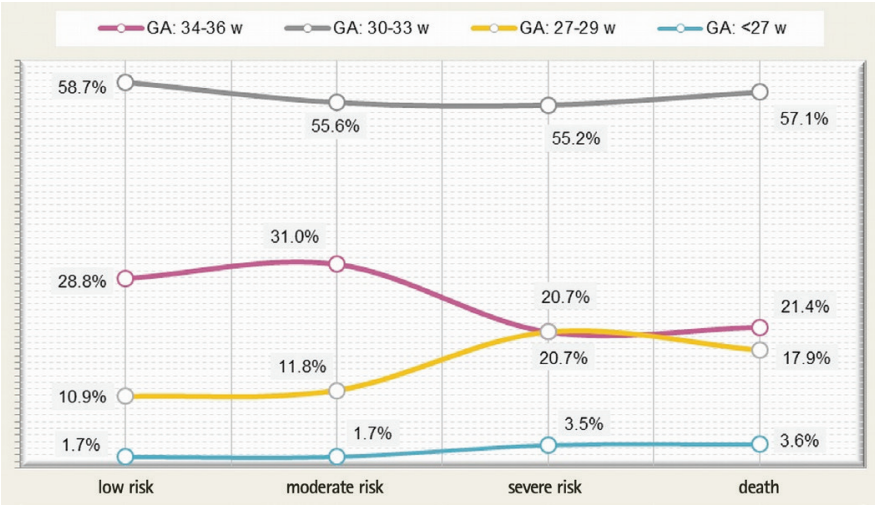

FIG. 3. Distribution of preterm infants according to the degree of risk and gestational age.

GA: gestational age
According to our "outcome score", 4.94\% of those with one evaluation and $0.43 \%$ with at least three received scores of "- 2 ". " -1 " received $70.37 \%$, those with one evaluation, $11.49 \%$ with least three evaluation and only $1.07 \%$ with regular evaluations, " 0 " score - $24.69 \%$ from those with one evaluation, $80 \%$ with minimum 3 evaluations and 56.12\% with regular evaluations, " +1 ", no one from those with one evaluation, $8.09 \%$ with least three evaluations and $42.45 \%$ with regular evaluations and " +2 ", no one from those with one and least three evaluation and $0.36 \%$ from those with regular evaluations (Table 2).

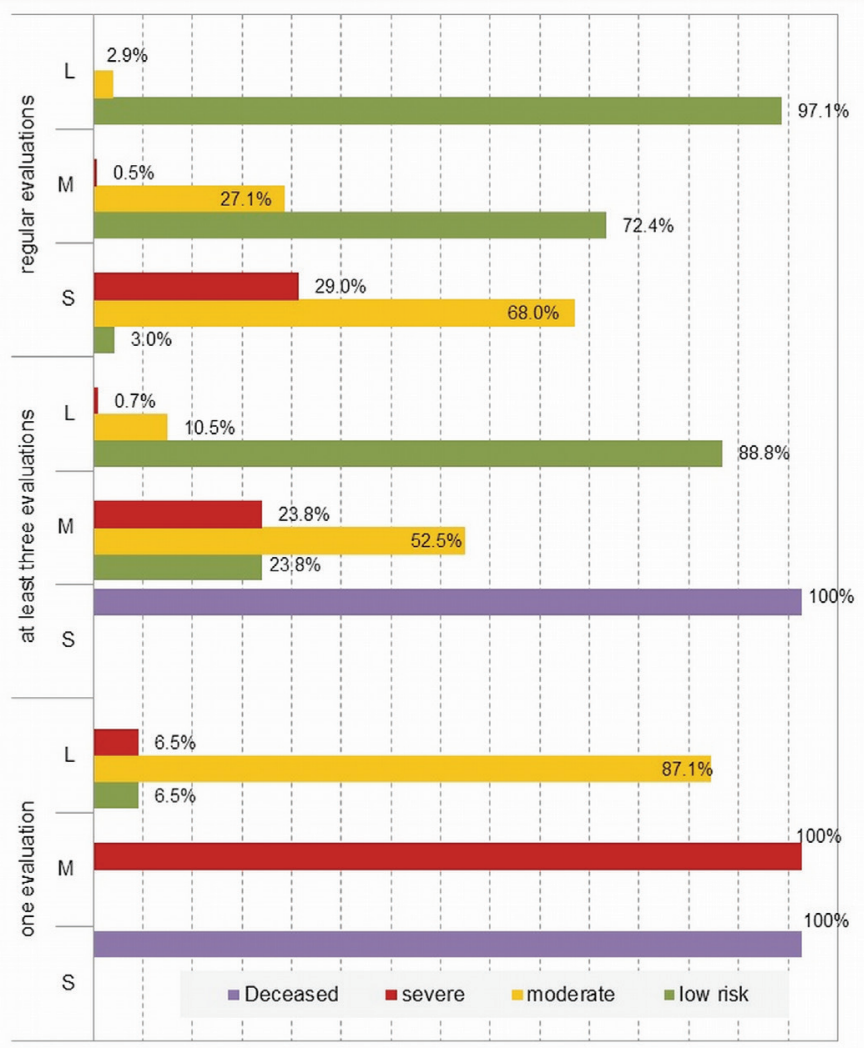

FIG. 4. Amiel-Tison examination and BSID II examination versus number of evaluations and degree of risk.

L: low; M: moderate; S: severe

TABLE 1. Evaluations by age and risk categories

\begin{tabular}{|c|c|c|c|c|c|}
\hline & Severe risk & Moderate risk & Low risk & & Deceased \\
\hline Discharge & $128(11.1 \%)$ & $482(41.7 \%)$ & $547(47.3 \%)$ & 1157 & \\
\hline $3 \mathrm{mo}$ & $101(8.7 \%)$ & $727(62.8 \%)$ & $329(28.4 \%)$ & 1157 & \\
\hline $6 \mathrm{mo}$ & $91(8.0 \%)$ & $676(59.4 \%)$ & $372(32.7 \%)$ & 1139 & 18 \\
\hline $9 \mathrm{mo}$ & $81(7.2 \%)$ & $514(45.5 \%)$ & $534(47.3 \%)$ & 1129 & 10 \\
\hline $12 \mathrm{mo}$ & $69(6.1 \%)$ & $472(41.8 \%)$ & $588(52.1 \%)$ & 1129 & \\
\hline $18 \mathrm{mo}$ & $66(5.8 \%)$ & $369(32.7 \%)$ & $69461.5 \%)$ & 1129 & \\
\hline $24 \mathrm{mo}$ & $58(5.1 \%)$ & $297(26.3 \%)$ & $774(68.6 \%)$ & 1129 & \\
\hline
\end{tabular}


TABLE 2. Outcome scores

\begin{tabular}{|c|c|c|c|c|c|c|}
\hline & \multicolumn{6}{|c|}{ OUTCOME } \\
\hline & $\begin{array}{c}-2 \\
(\mathrm{~L} \rightarrow \mathrm{S})\end{array}$ & $\begin{array}{c}-1 \\
(\mathrm{~L} \rightarrow \mathrm{M} \\
\mathrm{M} \rightarrow \mathrm{S})\end{array}$ & $\begin{array}{c}0 \\
(\mathrm{~L} \rightarrow \mathrm{L} \\
\mathrm{M} \rightarrow \mathrm{M} \\
\mathrm{S} \rightarrow \mathrm{S})\end{array}$ & $\begin{array}{c}+1 \\
(\mathrm{M} \rightarrow \mathrm{L} \\
\mathrm{S} \rightarrow \mathrm{M})\end{array}$ & $\begin{array}{c}+2 \\
(\mathrm{~S} \rightarrow \mathrm{L})\end{array}$ & Total \\
\hline \multirow[t]{2}{*}{ One evaluation } & 4 & 57 & 20 & 0 & 0 & 81 \\
\hline & $4.94 \%$ & $70.37 \%$ & $24.69 \%$ & $0.00 \%$ & $0.00 \%$ & \\
\hline \multirow[t]{2}{*}{ At least 3 evaluations } & 1 & 27 & 188 & 19 & 0 & 235 \\
\hline & $0.43 \%$ & $11.49 \%$ & $80.00 \%$ & $8.09 \%$ & $0.00 \%$ & \\
\hline \multirow[t]{2}{*}{ Regular evaluations } & 0 & 9 & 472 & 357 & 3 & 841 \\
\hline & $0.00 \%$ & $1.07 \%$ & $56.12 \%$ & $42.45 \%$ & $0.36 \%$ & \\
\hline Totals & 5 & 93 & 680 & 376 & 3 & 1157 \\
\hline
\end{tabular}

Correlation analysis demonstrated a significant association between the final category of risk at the end of the follow-up program and the degree of compliance $\left(\chi^{2}=618.70, p<0.001\right.$, $95 \% \mathrm{CI})$.

\section{DISCUSSION}

Since the early 1970s, it has been known that the neonatal neurological examination is valuable in predicting the future development of an infant. An abnormal result of the examination has been shown to correlate with an abnormal developmental outcome in preterm infants $(1,4,5)$. Monitoring the attainment of developmental milestones is essential for the early diagnosis of developmental disabilities, in order to institute early intervention and influence the quality of life for these patients.

According to the National Guidelines for Follow-up of the High-Risk Neonate, in Romania, this program is performed only in Level III maternity hospitals, up to the age of two years (chronologic for term infants and corrected for premature infants). In our neonatal intensive care unit, the followup program was implemented in 2006. The Amiel-Tison neurological examination test was performed at discharge, as close as possible to 40 weeks of gestation and the BSID II was performed during the two years of the follow-up program. Despite all the limitations and controversies surrounding this assessment tests, they do form an effective means of identifying infants with delayed development, so that early intervention can be started.

The region of Moldova has a low socio-economic status. Our maternity services extend throughout this entire area. The incidence of premature babies admitted in our unit increases from $19.27 \%$ to $27.23 \%$ over the studied period. This high percentage can be explained also by the fact that our hospital is a referral center for both high-risk pregnancies and sick preterm infants. The regional enrollment of a study population, despite the low socio-economic status of our region, points out a reasonable willingness of families to participate in the followup program (in our study, $72.7 \%$ came for regular evaluations). A reliable follow-up program depends on maintaining a high rate of participation.

Our study revealed a higher incidence of neurodevelopmental disabilities (29.9\%), compared with other data (18), probably due to the low social and economic status of the infants' families. Social and/or environmental characteristics of families and the mother-child interaction are predictive factors for later outcome $(22,23)$. Low socio-economic status was found to be an independent risk factor in the study conducted by Ruth et al. (24). The main limitation of our cohort study is the absence of these correlations. Although we acknowledge the major influence of factors such as initial condition, severity of neurologic injury or gestational age, we focused on the correlation between compliance to the follow-up program and neurologic risk outcome.

Gestational age is a decisive factor in motor, cognitive and behavioral development, in addition to intensive recovery therapy; the percentage of premature infants at $\mathrm{S}$ risk is inversely proportional to gestational age. Normal neurological assessment of preterm infants at 40 weeks corrected age, together with a gestational age of more than 34 weeks of gestation and no severe cerebral lesions, is associated with a significantly lower risk of suboptimal neurologic development at 2 years of corrected age (24).

In this study, the highest percentage $(42 \%)$ with L risk and regular evaluation was represented by infants with gestational age of 34-36 weeks. We might explain this by the fact that mothers were encouraged by the progressive favorable evolution of their infants. Meanwhile, only $2.4 \%$ of infants less than 27 weeks of gestation came for all 6 recommended evaluations. 
At the moment of hospital discharge, the neurological examination of high-risk premature infant is not decisive in predicting neurodevelopmental disabilities; however, certain abnormal findings are associated with abnormal neurologic acquisitions. By the age of 2 years, major handicaps such as gross motor disturbances and severe mental retardation will have become clear, but subtle neurological impairment, behavioral and learning difficulties, especially in former highrisk premature infants are often noticed at school age.

Due to the high rate of prematurity in Romania and the low socio-economic status of the majority of the population, an extended program of follow-up, at least up to 7 years of age, is badly needed, especially since some neurocognitive, executive and behavioral malfunctions can only be detected at school age and preterm birth has an effect into adulthood (25-30).

\section{CONCLUSION}

Gestational age remains the leading factor in including premature infants in a specific group of developmental risk. Our results demonstrate, importantly, that the category of risk can improve over time with repeated evaluations and specific therapy. S risk was $11.1 \%$ at discharge and $5.1 \%$ at 24 months corrected age (the end of the follow-up program). Because major developmental disabilities are likely to be found before 2 years of age, it can be assumed that the incidence of infants with high risk is not underestimated.

The present study highlights the strong correlation between regular evaluations and a decreased risk of developing neurological sequelae. This emphasizes the importance of follow-up programs for the early identification of the degree of risk, as well as the importance of involving the patients' families in order to improve the neurologic outcomes of their children.

Acknowledgements: The authors wish to thank the many families involved in the care of premature infants and would like to acknowledge their efforts to insure the best quality of life for their infants.

Conflict of Interest: No conflict of interest was declared by the authors.

\section{REFERENCES}

1. Costeloe KL, Hennessy EM, Haider S, Stacey F, Marlow N, Draper ES. Short term outcomes after extreme preterm birth in England: comparison of two birth cohorts in 1995 and 2006 (the EPICure studies). BMJ 2012;345:e7976.

2. Boyle EM, Poulsen G, Field DJ, Kurinczuk JJ, Wolke D, Alfirevic Z, et al. Effects of gestational age at birth on health outcomes at 3 and 5 years of age: population based cohort study. BMJ 2012;344:e896.
3. Kerstjens JM, Bocca-Tjeertes IF, de Winter AF, Reijneveld SA, Bos AF. Neonatal morbidities and developmental delay in moderately preterm-born children. Pediatrics 2012;130:e265-72.

4. Stoll BJ, Hansen NI, Bell EF, Shankaran S, Laptook AR, Walsh MC, et al; Eunice Kennedy Shriver National Institute of Child Health and Human Development Neonatal Research Network. Neonatal outcomes of extremely preterm infants from the NICHD Neonatal Research Network. Pediatrics 2010;126:443-56.

5. Platt MJ, Cans C, Johnson A, Surman G, Topp M, Torrioli MG, et al. Trends in cerebral palsy among infants of very low birth weight $(<1500$ grams $)$ or born prematurely ( $<32 \mathrm{wk}$.) in 16 European centres. Lancet. 2007;369:43-50.

6. Marlow N, Wolke D, Bracewell MA, Samara M; EPICure Study Group. Neurologic and developmental disability at six years of age after extremely preterm birth. N Engl J Med 2005;352:9-19.

7. Laroque B, Ancel PY, Marret S, Marchand L, Andre M, Arnaud C, et al ; EPIPAGE Study group. Neurodevelopmental disabilities and special care of 5-year-old children born before 33 weeks of gestation (the EPIPAGE study): a longitudinal cohort study. Lancet 2008;371:813-20.

8. Hack M. Adult outcomes of preterm children. J Dev Behav Pediatr 2009;30:460-70.

9. Bhutta AT, Cleves MA, Casey PH, Craddock MM, Anand KJS. Cognitive and behavioral outcomes of school-age children who were born preterm: a meta-analysis. JAMA 2002;288:728-37.

10. Steward AL, Rifkin L, Amess PN, Kirkbride V, Townsend JP, Miller DH, et al. Brain structure and neurocognitive and behavioural function in adolescents who were born very preterm. Lancet 1999;353:1653-7.

11. Stoll BJ, Hansen NI, Bell EF, Shankaran S, Laptook AR, Walsh MC, et al; Eunice Kennedy Shriver National Institute of Child Health and Human Development Neonatal Research Network. Neonatal outcomes of extremely preterm infants from the NICHD Neonatal Research Network. Pediatrics 2010;126:443-56.

12. Singh GK, Kenney MK, Reem M, Kogan MD, Lu MC. Mental Health Outcomes in US Children and Adolescents Born Prematurely or with Low Birthweight. Depress Res Treat 2013;2013:570743.

13. Taylor HG, Klein N, Minich NM, Hack M. Middle-school-age outcomes in children with very low birth weight. Child Dev 2000;71:1495-511.

14. Blencowe H, Cousens S, Chou D, Oestergaard M, Say L, Moller AB, et al. Born too soon: The global epidemiology of 15 million preterm births. Reprod Health 2013;10 Suppl 1:S2.

15. Blencowe H, Cousens S, Oestergaard MZ, Chou D, Moller AB, Narwal $\mathrm{R}$ et al. National, regional, and worldwide estimates of preterm birth rates in the year 2010 with time trends since 1990 for selected countries: a systematic analysis and implications. Lancet 2012;379:2162-72.

16. Mâțu E. Urmărirea nou-născutului cu risc pentru sechele neurologice și de dezvoltare. Asociația de Neonatologie din România, 2011. Colecția ghiduri clinice pentru neonatologie. Ghidul 13/revizia 03.12.2010. Ghiduri clinice - Ministerul Sănătății http://www.ghiduriclinice.ro/neonatologie-ghiduriclinice.php

17. Amiel-Tison C. Clinical assessment of the infant nervous system. In: Levene MI, Chervenak FA, Whittle M, editors. Fetal and Neonatal Neurology and Neurosurgery. London: Churchill Livingstone. 2001;99-120.

18. Leroux BG, N'Guyen The Tich S, Branger B, Gascoin G, Rouger V, Berlie I, et al. Neurological assessment of preterm infants for predicting neuromotor status at 2 years: results from the LIFT cohort. BMJ Open 2013;3.

19. Bayley N. Bayley Scales of Infant Development. 2nd ed. in: J Psychoed Assess 2000;18:185-95. http://www.pearsonclinical.com/childhood/ products/100000123/bayley-scales-of-infant-and-toddler-developmentthird-edition-bayley-iii.html

20. Aylward GP, Verhulst SJ, Bell S. Predictive utility of the BSID-II Infant Neurodevelopmental Screener (BINS) Risk Status Classifications. Dev Med Child Neurol J 1996;138(Suppl 74):26-36. 
21. Dezoete JA, MacArthur BA, Tuck B. Prediction of Bayley and Stanford Binet scores with a group of very low birth weight children. Child Care Health Dev 2003;29:367-72.

22. Bradley RH, Corwyn RF. Socioeconomic status and child development. Annu Rev Psychol 2002;53:371-99.

23. Wild KT, Betancourt LM, Brodsky NL. The effect of socioeconomic status on the language outcome of preterm infants at toddler age. Early Hum Dev 2013;89:743-6.

24. Ruth CA, Roos N, Hildes-Ripstein E, Brownell M. The influence of gestational age and socioeconomic status on neonatal outcomes in late preterm and early term gestation: a population based study. BMC Pregnancy Childbirth 2012;12:62.

25. Johnson S, Wolke D, Hennessy E, Smith R, Trikic R, Marlow N. Academic attainment and special educational needs in extremely preterm children at 11 years of age: the EPICure study. Arch Dis Child Fetal Neonatal Ed 2009;94:F283-9.
26. Litt JS, Gerry Taylor H, Margevicius S, Scluchtter M, Andreias L, Hack M. Academic achievement of adolescents born with extremely low birth weight. Acta Paediatr 2012;101:1240-5.

27. Beaino G, Khoshnood B, Kaminski M, Marret S, Pierrat V, Vieux R, et al ; EPIPAGE Study Group. Predictors of the risk of cognitive deficiency in very preterm infants: the EPIPAGE prospective cohort. Acta Paediatr 2011;100:370-8.

28. Aarnoudse-Moens CS, Weisglas-Kuperus N, van Goudoever JB, Oosterlaan J. Meta-analysis of neurobehavioral outcomes in very preterm and/or very low birth weight children. Pediatrics 2009;124:717-28.

29. Potijk MR, Kerstjens JM, Bos AF, Reijneveld SA, de Winter AF. Developmental delay in moderately preterm-born children with low socioeconomic status: risks multiply. J Pediatr 2013;163:1289-95.

30. Cserjesi R, VAN Braeckel KN, Timmerman M., Butcher PR, Kerstjens $\mathrm{JM}$, Reijneveld SA, et al. Patterns of functioning and predictive factors in children born moderately preterm or at term. Dev Med Child Neurol 2012;54:710-5. 